\title{
403 - Verbal fluency and spontaneous conversation in institutionalized older adults with and without cognitive impairment
}

Cristina G. Dumitrache; Laura Rubio, PhD; Nuria Calet; José Andrés González; Ian C. Simpson

Background: Several neurodegenerative conditions negatively impact linguistics skills. Despite this, many studies carried out with these kinds of patients either only include participants with initial stages of cognitive impairment either do not contemplate linguistic skills, or they do assess language in clinical or experimental settings. Due to it this study aims at investigating verbal fluency and spontaneous conversation abilities in a group of institutionalized Spanish older adults with and without cognitive impairment.

Method: The sample comprised 50 older adults who were residing in care homes from the province of Granada and with ages between 52 and 92 years old $(M=83.40, S D=7.18)$. The Mini Mental State Examination (MMSE), the Global Deterioration Scale, and the Short Form of the Boston Naming Test for Individuals with Aphasia were used to collect data. In order to analyze the differences in verbal fluency and in spontaneous conversation between participants ANOVA analysis were performed.

Results: Results showed that people without cognitive impairment or with initial stages of Parkinson's' disease showed a higher complexity in their spontaneous conversation and obtained higher scores in verbal fluency when compared with patients with Alzheimer's disease, and with people with cognitive impairment but without a clinical diagnose. No significant differences were found between participants in word or sentence repetitions tasks.

Conclusions: Language impairment in people with cognitive impairment has dramatic consequences, affecting people's communication and social interaction, their identity and autonomy thus language skills should be assessed in institutionalized older adults with cognitive impairment and interventions should be designed to maintain their linguistic abilities.

Keywords: cognitive impairment, dementia, institutionalized older adults, linguistic skills, verbal fluency 